J. Nonlinear Var. Anal. 5 (2021), No. 3, pp. 353-370

Available online at http://jnva.biemdas.com

https://doi.org/10.23952/jnva.5.2021.3.02

\title{
FREE BOUNDARY AND RETIREMENT BENEFITS PRICING IN A JUMP-DIFFUSION MODEL
}

\author{
BAOJUN BIAN ${ }^{1}$, CHAOYANG HAO $^{1}$, HONG-KUN XU ${ }^{2,3, *}$, QUAN YUAN ${ }^{1}$ \\ ${ }^{1}$ School of Mathematical Sciences, Tongji University, Shanghai 200092, China \\ ${ }^{2}$ School of Science, Hangzhou Dianzi University, Hangzhou 310018, China \\ ${ }^{3}$ College of Mathematics and Information Science, Henan Normal University, Xinxiang 453007, China
}

\begin{abstract}
The purpose of this paper is to give, by PDE argument, a pricing analysis for the defined benefits retirement pension plan that allows early retirement. The paid benefits on retirement depend on the time and the salary at that time which is assumed to follow a jump-diffusion process. Existence, uniqueness and regularity of the solution to an integro-differential parabolic variational inequality which models the retirement benefits pricing are proved. We also discuss the corresponding free boundary problem and deduce properties of the free boundary which corresponds to the optimal strategy to retirement.
\end{abstract}

Keywords. Free boundary; Integro-differential variational inequality; Retirement benefit; Jump-diffusion process; Optimal retirement strategy.

\section{INTRODUCTION}

Consider the following Cauchy problem of an integro-differential parabolic variational inequality

$$
\begin{aligned}
\min \{\mathscr{L} u-f, u-\varphi\} & =0, \quad \text { a.e. }(x, t) \in Q_{T} \\
u(x, 0) & =\varphi(x, 0), \quad x \in \mathbb{R}
\end{aligned}
$$

for a second linear parabolic integro-differential operator $\mathscr{L}$ (see (2.7) below) and a obstacle function $\varphi(x, t)$, where $Q_{T}=\mathbb{R} \times(0, T]$. In this paper, we will investigate the existence, uniqueness and regularity of the solution to the Cauchy problem (1.1) and discuss the properties of its related free boundary.

The variational inequality (1.1) models the financial valuation of a defined benefits retirement pension plan that allows early retirement in a jump-diffusion model. The related free boundary describes the optimal retirement strategy for the member of the pension plan. A defined benefits pension plan (DB in short) is a type of pension plan in which the benefits on retirement is predetermined by a formula based on the employee's salary history, age, duration of employment and some other personalized factors, rather than investment returns. It is different from any pension plan where payouts are somewhat dependent on the performance of the portfolio

\footnotetext{
${ }^{*}$ Corresponding author.

E-mail addresses: bianbj@tongji.edu.cn (B. Bian), haochaoyang@tongji.edu.cn (C. Hao), xuhk@ @du.edu.cn (H.K. Xu), candy5191@163.com (Q. Yuan).

Received March 6, 2021; Accepted April 7, 2021.
}

(C)2021 Journal of Nonlinear and Variational Analysis 
in which the contributions are invested. In a defined benefits plan the company sponsoring the plan is in charge of the portfolio management and bears the investment risk. The benefits are usually distributed through life annuities. In a life annuity, employees receive equal periodic benefit payments (monthly, quarterly, etc.) for the rest of their lives.

Option pricing techniques have been adopted for the financial valuation of retirement benefits. A number of researchers have made important contributions to this area. In [1], the method of "pricing contingent claims" is first time applied to price defined benefits plans with the type of "Greater of Benefits". In [2], the authors give a more in-depth and more general study on this type of pension plans. They establish existence and uniqueness of solutions to a variational inequality which is satisfied by the value of the retirement benefits and also study the properties of the free boundary which corresponds to the optimal time to retirement. Other related work can be found in $[3,4]$ and the references therein.

In the work mentioned above, the underlying salary follows a continuous Markov process. However, the salary process is not always continuous. Instead, it may jump up or down due to some unexpected reasons, such as a bonus for an excellent employee of the company or a penalty for bad behavior. Furthermore, in real world, it is possible that some sudden events occur, such as nature disasters, major political changes, and so on. These sudden events lead the economic environment to change abruptly. As a result, the movement of salary looks like a jump. The jump-diffusion model describes this phenomena rightly. This is similar to the pricing of option in jump-diffusion model. For option pricing under jump-diffusion model, many studies have been conducted to modify the Black-Scholes model. See $[5,6,7,8,9]$ and the references therein.

The purpose of our paper is to give a pricing analysis for the defined benefits retirement pension plan in a jump-diffusion model. To our knowledge, the retirement pension plan under jump-diffusion processes with a solid mathematical analysis has not appeared in the literature before. We use the jump-diffusion model to describe the dynamics of the underlying salary $S_{t}$, so that our parabolic operator $\mathscr{L}$ is a nonlocal operator. Our value function(solution of variational inequality) is unbounded. This is different from that in American put option. We will establish some new estimates and prove the existence, uniqueness and regularity of the solution to the variational inequality. We prove the continuity of the free boundary. In contrast of American put option in jump models, the payoff function depends time in our problem. We can not prove the monotone for free boundary and the differentiability of the free boundary is left as an open problem.

The rest of this paper is organized as follows. In Section 2, we establish the pricing equation for the defined benefits retirement pension plan with jump-diffusion processes. In Section 3, we prove the existence, uniqueness and regularity of the solutions of the variational inequality (1.1) by solving a related penalized problem. A key point in the ingredients is to derive the uniform estimates. In Section 4, we discuss the stopping and continuation regions. In the final section, Section 5, we analyze the continuity of the free boundary and also determine, from the financial point of view, the optimal retirement strategy for the member of the pension plan according to the free boundary. 


\section{PROBlem Formulation}

Assume that the dynamics of the salary process $\left(S_{t}\right)_{t \geq 0}$ follow the stochastic differential equation $(\mathrm{SDE})$

$$
\frac{d S_{t}}{S_{t}}=\theta d t+\sigma d B_{t}+d\left(\sum_{j=1}^{N_{t}} U_{j}\right)
$$

where $\theta, \sigma$ are positive constants known as the accrual rate and volatility, respectively. The process $\left(B_{t}\right)_{t \geq 0}$ is a Brownian motion. The process $\left(N_{t}\right)_{t \geq 0}$ is a Poisson process with intensity $\lambda$, and $\left(U_{j}\right)_{j \geq 1}$ is a sequence of square integrable, independent, identically distributed random variables, with values in $(-1,+\infty)$. The parameter $\lambda$ of the Poisson process represents the frequency of jumps, and the random variable $U_{j}$ represents the relative amplitude of jumps. Furthermore, we assume that the stochastic processes $\left(B_{t}\right)_{t \geq 0},\left(N_{t}\right)_{t \geq 0},\left(U_{j}\right)_{j \geq 1}$ are independent. Let $P(z)$ be the distribution function of $U_{1}$ and $k=\mathbb{E}\left[U_{1}\right]$; it then follows that

$$
\int_{-1}^{+\infty} d P(z)=1, \quad \int_{-1}^{+\infty} z d P(z)=k
$$

To derive the mathematical model for the value of the pension plan, we first adjust the salary process $\left(S_{t}\right)_{t \geq 0}$ to risk-neutral state. If jump occurs in the interval $[t, t+d t]$, set $d q_{t}=1$ with probability $\lambda d t$; otherwise, set $d q_{t}=0$ with probability $(1-\lambda d t)$. Let the random variable $U$ represent the relative jump amplitude having the same distribution function as $U_{1}$. We can rewrite (2.1) as

$$
\frac{d S_{t}}{S_{t}}=(\theta-\lambda k) d t+\sigma d B_{t}+U d q_{t}
$$

The payout function $\Phi$ on retirement is assumed to follow the assumption in [2], i.e., the larger of the two quantities: (a) an amount depending on the salary $S_{t}$ at the retirement time $t$; (b) a defined amount which is independent of the salary $S_{t}$. Let $T$ be the maturity of the retirement pension plan and $T_{0} \in(0, T)$, the early retirement time. Then the payoff function $\Phi(S, t)$ is given by

$$
\Phi(S, t)=\left(1-\frac{T-t}{T-T_{0}}\right)^{+} \max \{A, a S\}, \quad(S, t) \in \mathbb{R}^{+} \times[0, T],
$$

where $A$ is a defined amount mentioned in (b) and $a S$ is the amount mentioned in (a) with a positive constant $a$.

Following [2], also from the actuarial mathematics literature [10], the insurance benefits are paid from the retirement funds due to death or disability. Benefits are also paid if a member withdraws in order to transfer to another pension fund. We assume that the total benefits $B(S, t)=\mu S$. Denote by $r$ the risk-free interest rate and set $\rho=r+\mu$.

Denote by $\Pi_{[t, T]}$ the set of all stopping times for the process $S_{t}$, taking values in $[t, T]$. Then the financial value $V(S, t)$ of the pension plan can be characterized as

$$
V(S, t)=\sup _{\tau \in \Pi_{[t, T]}} \mathbb{E}\left[e^{-\rho(\tau-t)} \Phi\left(S_{\tau}, \tau\right)+\int_{t}^{\tau} e^{-\rho(\eta-t)} B\left(S_{\eta}\right) d \eta \mid S_{t}=S\right]
$$


where $S_{t}$ evolves according to (2.3). This optimal stopping time problem is related to the following variational inequality by Ito's formula (see [11] and [12])

$$
\begin{aligned}
\min \{\mathscr{M} V-B, V-\Phi\} & =0, \quad \text { a.e. }(S, t) \in \mathbb{R}^{+} \times(0, T], \\
V(S, T) & =\Phi(S, T), \quad S \in \mathbb{R}^{+}
\end{aligned}
$$

where $\mathscr{M}$ is a parabolic operator defined by

$$
\mathscr{M} V=-\left\{\frac{\partial V}{\partial t}+\frac{1}{2} \sigma^{2} S^{2} \frac{\partial^{2} V}{\partial S^{2}}+(\theta-\lambda k) S \frac{\partial V}{\partial S}-(\rho+\lambda) V+\lambda \mathbb{E}[V((1+U) S, t)]\right\} .
$$

It is also related to a parabolic free boundary problem. Let $\Gamma$ denote the boundary of the set $\{(S, t) \mid V-\Phi(S, t)>0, S \geq 0,0 \leq t \leq T\}$. Then the optimal strategy is to retire as soon as the path $t \rightarrow\left(S_{t}, t\right)$ hits $\Gamma$, which means that determining the free boundary $\Gamma$ is also very important in our valuation. If $V(S, t)$ is a $C^{1,0} \cap W_{p}^{2,1}$ strong solution of (2.6), then we have $\frac{\partial V}{\partial S}=\frac{\partial \Phi}{\partial S}$ on $\Gamma$, and $\mathscr{M} V \geq \mu S$ a.e. in $\mathbb{R}^{+} \times(0, T]$ (see [13]).

From the financial point of view, a rational member will choose to retire if and only if he gets the full value of the retirement benefits. Thus he will not select early retirement if $V(S, t)>$ $\Phi(S, t)$ which also implies that there holds the equation $\mathscr{M} V=B$.

Letting $x_{*}=\log \frac{A}{a}$ and using the substitutions

$$
S=e^{x+x_{*}}, u(x, t)=V(S, T-t), \varphi(x, t)=\Phi(S, T-t), N(z)=P\left(e^{z}-1\right),
$$

we find that the operator $\mathscr{M}$ induces another operator $\mathscr{L}$ given by

$$
\mathscr{L} u=\frac{\partial u}{\partial t}-\frac{\sigma^{2}}{2} \frac{\partial^{2} u}{\partial x^{2}}-\left(\theta-\lambda k-\frac{\sigma^{2}}{2}\right) \frac{\partial u}{\partial x}+(\rho+\lambda) u-\lambda \int_{-\infty}^{+\infty} u(x+z, t) d N(z)
$$

in which we have

$$
N(-\infty)=0, N(+\infty)=1, \int_{-\infty}^{+\infty} e^{z} d N(z)=1+k
$$

Later on, we shall need the operator $\mathscr{L}_{0}$ defined by

$$
\mathscr{L}_{0} u=\frac{\partial u}{\partial t}-\frac{\sigma^{2}}{2} \frac{\partial^{2} u}{\partial x^{2}}-\left(\theta-\lambda k-\frac{\sigma^{2}}{2}\right) \frac{\partial u}{\partial x}+(\rho+\lambda) u
$$

The payout function $\phi$ is now rewritten as

$$
\varphi(x, t)=A\left(1-\frac{t}{T-T_{0}}\right)^{+} \max \left\{e^{x}, 1\right\}, \quad(x, t) \in Q_{T} .
$$

Let $f(x)=B(S)=\mu e^{x+x_{*}}=\mu \frac{A}{a} e^{x}$. Then $u(x, t)$ solves the nonlocal parabolic integro-differential variational inequality (1.1).

\section{EXISTENCE AND UNIQUENESS FOR VARIATIONAL INEQUALITIES}

At first, we need a comparison principle for nonlocal parabolic integro-differential operators in unbounded domain. Let

$$
\overline{\mathscr{L}} u=\frac{\partial u}{\partial t}-a(x, t) \frac{\partial^{2} u}{\partial x^{2}}+b(x, t) \frac{\partial u}{\partial x}+c(x, t) u-\lambda \int_{-\infty}^{+\infty} G(x, z) u(x+z, t) d N(z) .
$$

Suppose that

$$
0<a(x, t) \leq a,|b(x, t)| \leq b, c(x, t) \geq c, \text { and } 0 \leq G(x, z) \leq e^{z}+1,
$$


where $a, b, c$ are positive constants such that

$$
c>\lambda \int_{-\infty}^{+\infty}\left(e^{z}+1\right) d N(z) .
$$

We need a technical condition

$$
\int_{-\infty}^{+\infty}\left(|z|^{2}+1\right) e^{2|z|} d N(z)<\infty
$$

Lemma 3.1. Let $D$ be a domain in $Q_{T}$ and $\partial_{p} D$ be the parabolic boundary of $D$. Suppose $u(x, t) \in C\left(\bar{Q}_{T}\right) \cap C^{2,1}(D)$ and $\overline{\mathscr{L}} u \geq 0$ in $D$. Furthermore, assume that $u(x, t)$ satisfies the boundary condition

$$
u(x, t) \geq 0,(x, t) \in Q_{T} \backslash D
$$

and the growth condition

$$
u(x, t) \geq-m\left(e^{x}+1\right), \quad(x, t) \in D,
$$

where $m$ is a positive constant. Then $u(x, t) \geq 0$ in $D$.

Proof. Let $D_{L}=D \cap\{|x|<L\}$ and consider the following function in $Q_{T}$

$$
w(x, t)=u(x, t)+\frac{m}{L^{2}}\left(x^{2}+1\right)\left(e^{x}+1\right) e^{K t},
$$

where $L, K$ are positive constants to be chosen. Simple computations yield

$$
\overline{\mathscr{L}} w \geq \frac{m}{L^{2}}\left(x^{2}+1\right)\left(e^{x}+1\right) e^{K t}\left[K-5 a-2 b+c-2 \lambda \int_{-\infty}^{+\infty}\left(z^{2}+1\right)\left(e^{z}+1\right)^{2} d N(z)\right] .
$$

Choosing $K$ large enough, we obtain $\overline{\mathscr{L}} w \geq 0$ in $Q_{T} \backslash D_{L}$.

From boundary conditions (3.3) and (3.4), we find that $w \geq 0$ on $\partial_{p} D_{L}$. We also claim that $w(x, t) \geq 0$ in $\bar{D}_{L}$. Indeed, if this is not true, we may assume that $w(x, t)$ attains its negative minimum at $\left(x_{0}, t_{0}\right) \in D_{L}$, which leads to

$$
\begin{aligned}
0 \leq \overline{\mathscr{L}} w\left(x_{0}, t_{0}\right) & \leq c\left(x_{0}, t_{0}\right) w\left(x_{0}, t_{0}\right)-\lambda \int_{-\infty}^{+\infty} G(x, z) w\left(x_{0}+z, t_{0}\right) d N(z) \\
& \leq\left[c\left(x_{0}, t_{0}\right)-\lambda \int_{-\infty}^{+\infty}\left(e^{z}+1\right) d N(z)\right] w\left(x_{0}, t_{0}\right)<0
\end{aligned}
$$

by (3.2) and the fact that $w\left(x_{0}, t_{0}\right) \leq w\left(x, t_{0}\right)$ for all $x \in \mathbb{R}$. This is a contradiction, therefore, $w(x, t) \geq 0$ in $\bar{D}_{L}$. Consequently, for each $(x, t) \in D$, we have

$$
u(x, t) \geq-\frac{m}{L^{2}}\left(x^{2}+1\right)\left(e^{x}+1\right) e^{K t}
$$

for any $L>0$. Letting $L \rightarrow \infty$, we show that $u(x, t) \geq 0$ in $D$.

Remark 3.1. In the proof of Lemma 3.1, we need the conditions $u(x, t) \in C\left(\bar{Q}_{T}\right)$ and (3.3) because of the nonlocal term. If domain $D$ is bounded, then we don not need condition (3.4). By use of Bony's maximum principle, we conclude that the condition $u(x, t) \in C\left(\bar{Q}_{T}\right) \cap C^{2,1}(D)$ can be replaced by the condition $u(x, t) \in C\left(\bar{Q}_{T}\right) \cap W_{p}^{2,1}(D)$ for some $p>3$. Furthermore, if $\left(x_{0}, t_{0}\right) \in \partial_{p} D$ and $\liminf _{(x, t) \rightarrow\left(x_{0}, t_{0}\right)} u(x, t) \geq 0$, then condition $u(x, t) \in C\left(\bar{Q}_{T}\right)$ can be replaced by $u(x, t) \in C\left(\bar{Q}_{T} \backslash\left(x_{0}, t_{0}\right)\right)$. 
To derive uniform estimates and existence, we will reformulate variational inequality (1.1). Define $h(x, t)=e^{K t}\left(e^{x}+1\right)$ with

$$
K>|\theta-\lambda k|+\lambda(1+k)
$$

and let

Then

$$
v=\frac{u}{h}, \quad \bar{\varphi}=\frac{\varphi}{h}, \quad \bar{f}=\frac{f}{h} .
$$

$$
\begin{gathered}
0 \leq \bar{\varphi}(x, t) \leq A, \quad-A \leq \bar{\varphi}_{x} \leq A(x \neq 0), \\
0 \leq \bar{f}, \quad \bar{f}_{x}, \quad\left|\bar{f}_{x x}\right| \leq \frac{\mu A}{a}
\end{gathered}
$$

and $\bar{\varphi}_{x x} \geq-3 A$ in the distribution sense. It turns out that (1.1) is reduced to the variational inequality

$$
\begin{aligned}
\min \{\overline{\mathscr{L}} v-\bar{f}, v-\bar{\varphi}\} & =0, \quad \text { a.e. }(x, t) \in Q_{T} \\
v(x, 0) & =\bar{\varphi}(x, 0), \quad x \in \mathbb{R},
\end{aligned}
$$

where the operator $\overline{\mathscr{L}}$ is defined by

$$
\begin{aligned}
\overline{\mathscr{L}} v:=\frac{\partial v}{\partial t}- & \frac{\sigma^{2}}{2} \frac{\partial^{2} v}{\partial x^{2}}-\left(\theta-\lambda k-\frac{\sigma^{2}}{2}+\sigma^{2} \frac{e^{x}}{e^{x}+1}\right) \frac{\partial v}{\partial x}+\left(\rho+\lambda+K+(\lambda k-\theta) \frac{e^{x}}{e^{x}+1}\right) v \\
& -\lambda \int_{-\infty}^{+\infty} \frac{e^{x+z}+1}{e^{x}+1} v(x+z, t) d N(z) .
\end{aligned}
$$

Note that, due to (3.5), the operator $\overline{\mathscr{L}}$ satisfies the conditions in Lemma 3.1, with $G(x, z)=$ $\frac{e^{x+z}+1}{e^{x}+1}$.

We work with the following approximating penalized problem as in $[2,6]$ to prove the existence of solutions to the integro-differential variational inequality (3.8) which is then used to prove the existence of solutions to (1.1).

For $\varepsilon>0$, define a penalty function $\beta_{\varepsilon}(s) \in C^{\infty}$ such that for some positive constant $C_{1}$ independent of $\varepsilon$,

$$
-A_{\varepsilon}(s+1) \leq \beta_{\varepsilon}(s) \leq 0, \quad \beta_{\varepsilon}(0) \geq-C_{1}, \quad \beta_{\varepsilon}^{\prime}(s) \geq 0, \quad \beta_{\varepsilon}^{\prime \prime}(s) \leq 0,
$$

and

$$
\lim _{\varepsilon \rightarrow 0} \beta_{\varepsilon}(s)=\left\{\begin{array}{cl}
0 & \text { if } s>0, \\
-\infty & \text { if } s<0 .
\end{array}\right.
$$

We also define a function $\varphi_{\varepsilon}(x, t) \in C^{2}$ satisfying

$$
\begin{aligned}
& \varphi_{\varepsilon}(x, t)=\varphi(x, t), \text { if }|x|>\varepsilon, \\
& \frac{\partial}{\partial x} \varphi_{\varepsilon} \geq 0, \frac{\partial^{2}}{\partial x^{2}} \varphi_{\varepsilon} \geq 0, \\
& \varphi_{\varepsilon}(x, t) \rightarrow \varphi(x, t), \text { if } \varepsilon \rightarrow 0 .
\end{aligned}
$$

Let $\bar{\varphi}_{\varepsilon}=\frac{\varphi_{\varepsilon}}{h}$. Then,

$$
0 \leq \bar{\varphi}_{\varepsilon} \leq A, \quad\left|\frac{\partial}{\partial x} \bar{\varphi}_{\varepsilon}\right| \leq A, \quad-3 A \leq \frac{\partial}{\partial x^{2}} \bar{\varphi}_{\varepsilon}
$$


To solve (3.8), we consider the following approximating penalized problem

$$
\begin{aligned}
\overline{\mathscr{L}} v_{\varepsilon, R}+\beta_{\varepsilon}\left(v_{\varepsilon, R}-\bar{\varphi}_{\varepsilon}\right) & =\bar{f} \text { in } Q_{T, R} \\
v_{\varepsilon, R} & =\bar{\varphi}_{\varepsilon} \quad \text { on } \partial_{p} Q_{T, R},
\end{aligned}
$$

where $Q_{T, R}=Q_{T} \cap\{|x|<R\}$. For the nonlocal term, we define $v_{\varepsilon, R}(x, t)=v_{\varepsilon, R}(R, t)$ for $x>R$ and $v_{\varepsilon, R}(x, t)=v_{\varepsilon, R}(-R, t)$ for $x<-R$. The linear growth of the penalty function $\beta_{\varepsilon}$ ensures the existence and uniqueness of solutions of (3.12). The proof follows from the standard fixed point theorem (see [6]).

Proposition 3.1. For every fixed $\varepsilon$ and $R$, there exists a unique solution $v_{\varepsilon, R} \in C^{\infty}\left(Q_{T, R}\right)$ for the penalized problem (3.12).

Next we derive uniform estimates for $v_{\varepsilon, R}$.

Lemma 3.2. There exists a positive constant $C_{2}$ such that, for any $\varepsilon, R>0$,

$$
0 \leq v_{\varepsilon, R}(x, t) \leq C_{2}
$$

Proof. It is evident that $v_{\varepsilon, R}(x, t) \geq 0$. Let $w(x, t)=C_{2}-v_{\varepsilon, R}(x, t)$. Choose $K$ as in (3.5) and $C_{2}$ large enough so that

$$
\begin{aligned}
\overline{\mathscr{L}} w+\beta_{\varepsilon}^{\prime} w & \geq(K+\rho+\lambda-\theta) C_{2}-C_{1}-\bar{f} \geq 0, \quad(x, t) \in Q_{T, R}, \\
w & \geq \bar{\varphi}_{\varepsilon}, \quad(x, t) \in \partial_{p} Q_{T, R},
\end{aligned}
$$

where $C_{1}$ satisfies (3.10). Note that $w(x, t) \geq 0$ in $Q_{T} \backslash Q_{T, R}$. It follows that $v_{\varepsilon, R}(x, t) \leq C_{2}$ by the comparison principle (Lemma 3.1).

Next we discuss the bound of the function $\beta_{\varepsilon}\left(v_{\varepsilon, R}-\bar{\varphi}_{\varepsilon}\right)$, which requires to be independent of $\varepsilon, R$.

Lemma 3.3. There exists a positive constant $C_{3}$ such that, for any $\varepsilon, R>0$,

$$
0 \leq-\beta_{\varepsilon}\left(v_{\varepsilon, R}-\bar{\varphi}_{\varepsilon}\right) \leq C_{3}, \quad(x, t) \in Q_{T, R} .
$$

Proof. Letting $w(x, t)=\beta_{\varepsilon}\left(v_{\varepsilon, R}-\bar{\varphi}_{\varepsilon}\right)$, we have $w \leq 0$. Let $\gamma=w\left(x_{0}, t_{0}\right)$, be the minimum value of $w(x, t)$ on $\bar{Q}_{T, R}$. If $\gamma \geq \beta_{\varepsilon}(0)$, then $\gamma \geq-C_{1}$ by (3.10). Assume $\gamma<\beta_{\varepsilon}(0)<0$. Then $\left(x_{0}, t_{0}\right) \notin \partial_{p} Q_{T, R}$ and $v_{\varepsilon, R}-\bar{\varphi}_{\varepsilon}$ attains its negative minimum at $\left(x_{0}, t_{0}\right)$ by the monotonicity of $\beta_{\varepsilon}$. Hence, taking $K>\lambda \int_{-\infty}^{+\infty}\left(e^{z}+1\right) d N(z)$,

$$
\overline{\mathscr{L}}\left(v_{\varepsilon, R}-\bar{\varphi}_{\varepsilon}\right)\left(x_{0}, t_{0}\right) \leq 0 .
$$

It turns out by (3.11) and (3.12) that

$$
\gamma \geq \bar{f}\left(x_{0}\right)-\overline{\mathscr{L}} \bar{\varphi}_{\varepsilon}\left(x_{0}, t_{0}\right) \geq-C_{3},
$$

where $C_{3}$ is independent of $\varepsilon, R$; consequently, $-\beta_{\varepsilon}\left(v_{\varepsilon, R}-\bar{\varphi}_{\varepsilon}\right) \leq C_{3}$.

Let $R_{0}>0, R>R_{0}+1$. We see that $\bar{\varphi}_{\varepsilon}$ is uniformly semi-convex from (3.6), (3.7) and (3.11). Applying the standard regularity theory of PDEs and proceeding as in $[2,6,8,13]$, we get the following estimates. 
Lemma 3.4. There exist positive constants $\alpha$ and $C_{4}$ such that

$$
\left\|v_{\varepsilon, R}\right\|_{C^{\alpha, \frac{\alpha}{2}}\left(Q_{T, R_{0}}\right)}, \quad\left\|\left(v_{\varepsilon, R}\right)_{x}\right\|_{C^{0}}\left(Q_{T, R_{0}}\right) \leq C_{4} .
$$

Furthermore, for any $p>1$ and any small $\eta>0$, there exists a positive constant $D_{\eta}$ such that

$$
\left\|v_{\varepsilon, R}\right\|_{W_{p}^{2,1}\left(Q_{T, R_{0}} \backslash B_{\eta}^{+}(0)\right)} \leq D_{\eta}, \quad\left|\left(v_{\varepsilon, R}\right)_{t}\right|, \quad\left|\left(v_{\varepsilon, R}\right)_{x x}\right| \leq D_{\eta}, \quad \text { in } \quad Q_{T, R_{0}} \backslash B_{\eta}^{+}(0),
$$

where $B_{\eta}^{+}(0)=\left\{(x, t) \mid x^{2}+t^{2}<\eta^{2}, t \geq 0\right\}$.

We conclude this section with the result below.

Theorem 3.1. There exists a unique strong solution $u \in C\left(\bar{Q}_{T}\right) \cap W_{p, l o c}^{2,1}\left(Q_{T} \backslash(0,0)\right)$ (any $\left.p>3\right)$ for the variational inequality (1.1) satisfying the properties

$$
0 \leq u(x, t) \leq C_{2} e^{K t}\left(e^{x}+1\right),\left|u_{x}(x, t)\right| \leq \bar{C}_{4} e^{K t}\left(e^{x}+1\right),(x, t) \in Q_{T}
$$

and

$$
\left|u_{t}(x, t)\right|,\left|u_{x x}(x, t)\right| \leq \overline{\bar{D}_{\eta}} e^{K t}\left(e^{x}+1\right),(x, t) \in Q_{T} \backslash B_{\eta}^{+}(0) .
$$

Proof. By the standard convergence theory and Lemma 3.4, we can show, as in $[2,6,8,13]$, that there exists a strong solution $v \in C\left(\bar{Q}_{T}\right) \cap W_{p, l o c}^{2,1}\left(Q_{T} \backslash(0,0)\right)$ (any $p>3$ ) of the variational inequality (3.8) with the properties

$$
0 \leq v(x, t) \leq C_{2},\left|v_{x}(x, t)\right| \leq C_{4},(x, t) \in Q_{T}
$$

and

$$
\left|v_{t}(x, t)\right|,\left|v_{x x}(x, t)\right| \leq D_{\eta},(x, t) \in Q_{T} \backslash B_{\eta}^{+}(0) .
$$

Hence we get the existence and estimates for solution of (1.1).

To prove the uniqueness of solutions, we assume that $u_{1}, u_{2} \in C\left(\overline{Q_{T}}\right) \cap W_{p}^{2,1}\left(Q_{T} \backslash(0,0)\right)$ $(p>3)$ are two solutions of (1.1). Let $D=\left\{(x, t) \mid u_{1}(x, t)<u_{2}(x, t)\right\}$ and $w=u_{1}-u_{2}$. Noting that $u_{2}(x, t)>u_{1}(x, t) \geq \varphi(x, t)$ for $(x, t) \in D$, it then follows

$$
\begin{aligned}
\mathscr{L} w & =\mathscr{L} u_{1}-\mathscr{L} u_{2}=\mathscr{L} u_{1}-f \geq 0, \text { in } D, \\
w & \geq 0, \text { on } Q_{T} \backslash D, \\
|w| & \leq C\left(e^{x}+1\right) .
\end{aligned}
$$

By Lemma 3.1 (see Remark 3.1), we deduce that $w \geq 0$ in $D$ and, therefore, $u_{1}(x, t) \geq u_{2}(x, t)$ for all $(x, t) \in Q_{T}$. Since the reverse inequality $u_{2} \geq u_{1}$ can similarly proved, we conclude that $u_{1} \equiv u_{2}$, which completes the proof of the uniqueness.

\section{Stopping REGion}

To determine the optimal time to retire for a member of the pension plan, it is important to find the free boundary related to the variational inequality (1.1). As in [2], to analyze the properties of the free boundary, we study the stopping region $\mathscr{S}$, in which the retiring member will receive the full value of the retirement benefits. Here the stopping region is defined by

$$
\mathscr{S}:=\{(x, t) \mid t>0, u(x, t)=\varphi(x, t)\}
$$

and the continuation region is defined by

$$
\mathscr{C}:=\{(x, t) \mid t>0, u(x, t)>\varphi(x, t)\} .
$$


The free boundary $\Gamma$ is defined by

$$
\Gamma:=\partial \mathscr{C} \cap\{0<t \leq T\} .
$$

We will investigate these sets in this section.

Firstly, we discuss some properties of the solution of variational inequality (1.1).

Lemma 4.1. For any $t \in(0, T]$, there holds

$$
u(0, t)>\varphi(0, t) .
$$

Proof. Observe that $u \geq \varphi$ and $u_{x}$ is continuous in $x$. It is clear that $u(x, t)>0=\varphi(x, t)$ for $t \in\left[T-T_{0}, T\right]$. Suppose that (4.1) failed to hold; then there would be some $t_{0} \in\left(0, T-T_{0}\right)$ such that $u\left(0, t_{0}\right)=\varphi\left(0, t_{0}\right)$. It turns out that

$$
u_{x}\left(0^{-}, t_{0}\right) \leq \varphi_{x}\left(0^{-}, t_{0}\right)=0, u_{x}\left(0^{+}, t_{0}\right) \geq \varphi_{x}\left(0^{+}, t_{0}\right)=A\left(1-\frac{t}{T-T_{0}}\right)>0 .
$$

This however contradicts the continuity of $u_{x}$ and the proof of Lemma 4.1 is finished.

Lemma 4.1 implies that $\Gamma \cap\{x=0\}=\emptyset$ and the free boundary can not touch $\{x=0\}$.

The stopping region and free boundary depend on the behavior of $\mathscr{L} \varphi-f$.

Lemma 4.2. Let

$$
\tilde{\mathscr{P}}:=\left\{(x, t) \mid \mathscr{L}_{0}(\varphi-u)(x, t) \geq 0\right\}, \mathscr{P}:=\{(x, t) \mid(\mathscr{L} \varphi-f)(x, t) \geq 0\} .
$$

Then $\mathscr{S} \subset \tilde{\mathscr{P}} \subset \mathscr{P}$.

Proof. Noting that $u \geq \varphi$ in $Q_{T}$ and $u=\varphi$ in $\mathscr{S}$, we conclude that $u-\varphi$ attains its minimum at $(x, t)$ for any $(x, t) \in \mathscr{S}$. It gives $\mathscr{L}_{0}(\varphi-u)(x, t) \geq 0$ for a.e. $(x, t) \in \mathscr{S}$ by Bony's maximum principle. Noting $u-\varphi \geq 0$ and $\mathscr{L} u \geq f$ a.e. in $Q_{T}$, we have, for a.e. $(x, t) \in \mathscr{S}$,

$$
\begin{aligned}
\mathscr{L} \varphi & =\mathscr{L}_{0} \varphi-\lambda \int_{-\infty}^{+\infty} \varphi(x+z, t) d N(z) \\
& \geq \mathscr{L}_{0} u-\lambda \int_{-\infty}^{+\infty} u(x+z, t) d N(z)=\mathscr{L} u \geq f .
\end{aligned}
$$

The conclusion follows.

It is clear that $\mathscr{L} \varphi(x, t)=0$ for $t \in\left[T-T_{0}, T\right]$ and, therefore, $\mathscr{P} \subset \mathbb{R} \times\left[0, T-T_{0}\right)$. For $t \in\left[0, T-T_{0}\right)$, a simple computation show that

$$
\mathscr{L}_{0} \varphi= \begin{cases}A\left[(\rho+\lambda)\left(1-\frac{t}{T-T_{0}}\right)-\frac{1}{T-T_{0}}\right], & x<0 \\ A\left[(\rho-\theta+\lambda+\lambda k)\left(1-\frac{t}{T-T_{0}}\right)-\frac{1}{T-T_{0}}\right] e^{x}, & x>0\end{cases}
$$

and

$$
\int_{-\infty}^{+\infty} \varphi(x+z, t) d N(z)=A\left(1-\frac{t}{T-T_{0}}\right)\left[1+\int_{-x}^{+\infty}\left(e^{x+z}-1\right) d N(z)\right] .
$$

Define the sets

$$
\begin{array}{cc}
\mathscr{P}_{-}:=\mathscr{P} \cap\{x<0\}, \mathscr{P}_{+}:=\mathscr{P} \cap\{x>0\}, \\
\mathscr{S}_{-}:=\mathscr{S} \cap\{x<0\}, \mathscr{S}_{+}:=\mathscr{S} \cap\{x>0\}, \\
\Gamma_{-}:=\Gamma \cap\{x<0\}, \Gamma_{+}:=\Gamma \cap\{x>0\} .
\end{array}
$$

We will study these sets and the free boundaries for $x<0$ and $x>0$, respectively. 
4.1. The Stopping Region in $\{x<0\}$. We discuss the stopping region $\mathscr{S}_{-}$in $\{x<0\}$. In this case, we obtain from (4.3) and (4.4)

$$
\frac{1}{A}(\mathscr{L} \varphi-f)=\left(1-\frac{t}{T-T_{0}}\right)\left[\rho-\lambda \int_{-x}^{+\infty}\left(e^{x+z}-1\right) d N(z)\right]-\frac{1}{T-T_{0}}-\frac{\mu}{a} e^{x} .
$$

Therefore, we get

$$
\mathscr{P}_{-}=\left\{(x, t) \mid\left(1-\frac{t}{T-T_{0}}\right)\left[\rho-\lambda \int_{-x}^{+\infty}\left(e^{x+z}-1\right) d N(z)\right] \geq \frac{1}{T-T_{0}}+\frac{\mu}{a} e^{x}\right\} .
$$

Define the function

$$
l(t):=\frac{1}{1-\frac{t}{T-T_{0}}}, \quad t \in\left[0, T-T_{0}\right) .
$$

It is clear that

$$
l^{\prime}(t)>0, l(0)=1, l\left(\left(T-T_{0}\right)^{-}\right)=+\infty .
$$

Let

$$
g(x):=\frac{\rho-\lambda \int_{-x}^{+\infty}\left(e^{x+z}-1\right) d N(z)}{\frac{1}{T-T_{0}}+\frac{\mu}{a} e^{x}}, x<0 .
$$

We have

$$
g^{\prime}(x)<0, g(-\infty)=\frac{\rho}{\frac{1}{T-T_{0}}}, g(0)=\frac{\rho-\lambda \int_{0}^{+\infty}\left(e^{z}-1\right) d N(z)}{\frac{1}{T-T_{0}}+\frac{\mu}{a}} .
$$

Hence the function

$$
m(t):=g^{-1}(l(t))
$$

is strictly decreasing. We have

$$
\mathscr{P}_{-}=\{(x, t) \mid l(t) \leq g(x), t>0\}=\{(x, t) \mid x \leq m(t), t>0\},
$$

and

$$
\partial \mathscr{P}_{-} \cap\{t>0\}=\{(x, t) \mid l(t)=g(x), t>0\}=\{(x, t) \mid x=m(t), t>0\} .
$$

In view of $l(t)>1, t>1$, we get

$$
\rho-\frac{1}{T-T_{0}} \leq 0
$$

Then

$$
g(x)<g(-\infty)=\frac{\rho}{\frac{1}{T-T_{0}}} \leq 1
$$

and

$$
\mathscr{P}_{-}=\{(x, t) \mid l(t) \leq g(x)\}=\emptyset .
$$

Therefore, $\mathscr{S}_{-}=\emptyset$, i.e., there is no free boundary in $x<0$ in the case of (4.9).

If

$$
0<\rho-\frac{1}{T-T_{0}}<\frac{\mu}{a}+\lambda \int_{0}^{+\infty}\left(e^{z}-1\right) d N(z),
$$

then

$$
g(-\infty)=\frac{\rho}{\frac{1}{T-T_{0}}}>1, g(0)=\frac{\rho-\lambda \int_{0}^{+\infty}\left(e^{z}-1\right) d N(z)}{\frac{1}{T-T_{0}}+\frac{\mu}{a}}<1 .
$$

This implies that there exists a unique point $x^{-}<0$ such that

$$
g\left(x^{-}\right)=1, g(x)>1, x \in\left(-\infty, x^{-}\right),
$$


which implies that

$$
\mathscr{P}_{-}=\left\{(x, t) \mid l(t) \leq g(x), x \in\left(-\infty, x^{-}\right)\right\}
$$

and

$$
\begin{aligned}
\partial \mathscr{P}_{-} \cap\{t>0\} & =\{(x, t) \mid l(t)=g(x), t>0\} \\
& =\left\{(x, t) \mid t=m^{-1}(x), x \in\left(-\infty, x^{-}\right)\right\}
\end{aligned}
$$

is a strictly decreasing curve. In this case, $\left(x^{-}, 0\right)$ is the starting point of $\mathscr{P}_{-}$and

$$
\sup \left\{t \mid \exists x,(x, t) \in \mathscr{P}_{-}\right\}=T-T_{0}-\frac{1}{\rho} .
$$

We denote $\tau_{1}=T-T_{0}-\frac{1}{\rho}$. Then,

$$
\partial \mathscr{P}_{-} \cap\{t>0\}=\left\{(x, t) \mid x=m(t), t \in\left(0, \tau_{1}\right)\right\}
$$

and $m(0)=x^{-}, m\left(\tau_{1}^{-0}\right)=-\infty$. If

$$
\rho-\frac{1}{T-T_{0}}-\frac{\mu}{a}-\lambda \int_{0}^{+\infty}\left(e^{z}-1\right) d N(z) \geq 0,
$$

then

$$
g(0)=\frac{\rho-\lambda \int_{0}^{+\infty}\left(e^{z}-1\right) d N(z)}{\frac{1}{T-T_{0}}+\frac{\mu}{a}} \geq 1
$$

This implies that

$$
\mathscr{P}_{-}=\{(x, t) \mid l(t) \leq g(x), x \in(-\infty, 0)\}
$$

and

$$
\begin{aligned}
\partial \mathscr{P}_{-} \cap\{t>0\} & =\{(x, t) \mid l(t)=g(x), t>0\} \\
& =\left\{(x, t) \mid t=m^{-1}(x), x \in(-\infty, 0)\right\}
\end{aligned}
$$

is a strictly decreasing curve. In this case, $(0,0)$ is the starting point of $\mathscr{P}_{-}$and

$$
\sup \left\{t \mid \exists x,(x, t) \in \mathscr{P}_{-}\right\}=\tau_{1} .
$$

We have

$$
\partial \mathscr{P}_{-} \cap\{t>0\}=\left\{(x, t) \mid x=m(t), t \in\left(0, \tau_{1}\right)\right\}
$$

and $m(0) \geq 0, m\left(\tau_{1}^{-0}\right)=-\infty$.

Lemma 4.3. The stoping region $\mathscr{S}_{-} \neq \emptyset$ if (4.10) or (4.11) holds.

Proof. Assume (4.10) or (4.11). If $\mathscr{S}_{-}=\emptyset$, then $u(x, t)-\varphi(x, t)>0$ in $\{x<0, t>0\}$ and $u \in C^{2,1}$ is a classical solution of $\mathscr{L} u=f$ in $\{x<0, t \geq 0\}$ with initial condition $u(x, 0)=$ $\varphi(x, 0)$ for all $x$. Noting that $\mathscr{L} u(x, 0)=f(x)$ and $(u-\varphi)(x, 0)=0$ for $x<0$, we deduce that $(u-\varphi)_{t}(x, 0)=(f-\mathscr{L} \varphi)(x, 0)<0$ for $x<x^{-}$. Hence $u(x, t)-\varphi(x, t)<0$, provided $t$ is sufficiently small. This is a contradiction. 
4.2. The Stopping Region in $\{x>0\}$. Analogously to the procedures in subsection 4.1, we analyze the boundary of the stopping region $\mathscr{S}_{+}$in $\{x>0\}$. In this case, we obtain from (4.3) and (4.4)

$$
\begin{aligned}
\frac{1}{A e^{x}}(\mathscr{L} \varphi-f)=( & \left.1-\frac{t}{T-T_{0}}\right)\left[\rho+\lambda+\lambda k-\theta-\lambda e^{-x}-\lambda \int_{-x}^{+\infty}\left(e^{z}-e^{-x}\right) d N(z)\right] \\
& -\frac{1}{T-T_{0}}-\frac{\mu}{a}
\end{aligned}
$$

Therefore,

$$
\begin{array}{r}
\mathscr{P}_{+}=\left\{(x, t) \mid\left(1-\frac{t}{T-T_{0}}\right)\left[\rho+\lambda+\lambda k-\theta-\lambda e^{-x}\right.\right. \\
\left.\left.-\lambda \int_{-x}^{+\infty}\left(e^{z}-e^{-x}\right) d N(z)\right] \geq \frac{1}{T-T_{0}}+\frac{\mu}{a}\right\}
\end{array}
$$

Letting

$$
h(x)=\frac{\rho+\lambda+\lambda k-\theta-\lambda e^{-x}-\lambda \int_{-x}^{+\infty}\left(e^{z}-e^{-x}\right) d N(z)}{\frac{1}{T-T_{0}}+\frac{\mu}{a}}, x>0,
$$

we find

$$
h^{\prime}(x)>0, h(\infty)=\frac{\rho-\theta}{\frac{1}{T-T_{0}}+\frac{\mu}{a}}, h(0)=\frac{\rho-\theta-\lambda \int_{-\infty}^{0}\left(1-e^{z}\right) d N(z)}{\frac{1}{T-T_{0}}+\frac{\mu}{a}} .
$$

Then we have

$$
\mathscr{P}_{+}=\{(x, t) \mid l(t) \leq h(x)\}
$$

and

$$
\partial \mathscr{P}_{+} \cap\{t>0\}=\{(x, t) \mid l(t)=h(x), t>0\}=\left\{(x, t) \mid x=h^{-1}(l(t)), t>0\right\}
$$

is a strictly increasing curve.

If

$$
\rho-\frac{1}{T-T_{0}}-\frac{\mu}{a}-\theta \leq 0
$$

then

$$
h(x)<h(\infty)=\frac{\rho-\theta}{\frac{1}{T-T_{0}}+\frac{\mu}{a}} \leq 1 .
$$

Hence

$$
\mathscr{P}_{+}=\{(x, t) \mid l(t) \leq g(x)\}=\emptyset
$$

and $\mathscr{S}_{+}=\emptyset$, that is, there is no free boundary in $x>0$ in the case of (4.13).

If

$$
0<\rho-\frac{1}{T-T_{0}}-\frac{\mu}{a}-\theta<\lambda \int_{-\infty}^{0}\left(1-e^{z}\right) d N(z)
$$

then

$$
h(\infty)=\frac{\rho-\theta}{\frac{1}{T-T_{0}}+\frac{\mu}{a}}>1, h(0)=\frac{\rho-\theta-\lambda \int_{-\infty}^{0}\left(1-e^{z}\right) d N(z)}{\frac{1}{T-T_{0}}+\frac{\mu}{a}}<1 .
$$

This implies that there exists a unique point $x^{+} \in(0, \infty)$ such that

$$
h\left(x^{+}\right)=1, h(x)>1, x \in\left(x^{+}, \infty\right) .
$$


This in turns implies that

$$
\mathscr{P}_{+}=\left\{(x, t) \mid l(t) \leq h(x), x \in\left(x^{+}, \infty\right)\right\}
$$

and that

$$
\partial \mathscr{P}_{+} \cap\{t>0\}=\{(x, t) \mid l(t)=h(x), t>0\}=\left\{(x, t) \mid t=l^{-1}(h(x)), x \in\left(x^{+}, \infty\right)\right\}
$$

is a strictly decreasing curve. In this case, $\left(x^{+}, 0\right)$ is the starting point of $\mathscr{P}_{+}$and

$$
\sup \left\{t \mid \exists x,(x, t) \in \mathscr{P}_{+}\right\}=T-T_{0}-\frac{1+\frac{\mu}{a}\left(T-T_{0}\right)}{\rho-\theta} .
$$

We denote $\tau_{2}=T-T_{0}-\frac{1+\frac{\mu}{a}\left(T-T_{0}\right)}{\rho-\theta}$.

If

$$
\rho-\frac{1}{T-T_{0}}-\frac{\mu}{a}-\theta \geq \lambda \int_{-\infty}^{0}\left(1-e^{z}\right) d N(z)
$$

then

$$
h(0)=\frac{\rho-\theta-\lambda \int_{-\infty}^{0}\left(1-e^{z}\right) d N(z)}{\frac{1}{T-T_{0}}+\frac{\mu}{a}} \geq 1 .
$$

Consequently,

$$
\mathscr{P}_{+}=\{(x, t) \mid l(t) \leq g(x), x \in(0, \infty)\}
$$

and

$$
\partial \mathscr{P}_{+} \cap\{t>0\}=\{(x, t) \mid l(t)=g(x), t>0\}=\left\{(x, t) \mid t=l^{-1}(g(x)), x \in(0, \infty)\right\}
$$

is a strictly increasing curve. In this case, $(0,0)$ is the starting point of $\mathscr{P}_{+}$and

$$
\sup \left\{t \mid \exists x,(x, t) \in \mathscr{P}_{+}\right\}=\tau_{2} .
$$

We can obtain the same results for $\mathscr{S}_{+}$as in Lemma 4.3.

As a conclusion, we have the result below.

Theorem 4.1. There is no left free boundary in the case of (4.9) which implies that it is never sensible for a member to retire early. There exists one left free boundary in the other two cases (4.10) and (4.11). There is no right free boundary in the case (4.13) which implies that it is never sensible for a member to retire early. There exists one right free boundary in the other two cases (4.14) and (4.15).

\section{Properties of the Free Boundary}

5.1. Representation of the free boundary. We discuss the representation of left free boundary $\Gamma_{-}$.

Theorem 5.1. There holds

$$
(u-\varphi)_{x} \geq 0, \text { in } Q_{T} \cap\{x<0\} .
$$


Proof. We firstly prove

$$
u_{x}(x, t) \geq 0 \quad \text { in } Q_{T} .
$$

Letting $w=u_{x}$ and $\mathscr{C}=\{(x, t) \mid u(x, t)>\varphi(x, t), t>0\}$, we have $w=u_{x}=\varphi_{x} \geq 0$ for $(x, t) \in$ $Q_{T} \backslash(\mathscr{C} \cup(0,0)), \liminf _{(x, t) \rightarrow(0,0)} w(x, t) \geq 0$ and $\mathscr{L} w=f_{x}>0$ in $\mathscr{C}$. Consequently, it follows from Lemma 3.1 that

$$
w=u_{x}(x, t) \geq 0 \quad \text { in } \mathscr{C} .
$$

Therefore, $u_{x}(x, t) \geq 0$ in $Q_{T}$. Noting that $\varphi_{x}=0$ in $\{x<0\}$ we deduce (5.1).

Theorem 5.1 implies that if the point $(\bar{x}, \bar{t})$ belongs to the left stopping region $\mathscr{S}_{-}$, then the interval $\{(x, \bar{t}) \mid x \leq \bar{x}\}$ also belongs to the stopping region $\mathscr{S}$. Therefore, we can define

$$
s(t)=\sup \{x \mid x<0, u(x, t)=\varphi(x, t)\}, 0<t<\tau_{1},
$$

and the left free boundary

$$
\Gamma_{-}=\left\{(s(t), t) \mid 0<t<\tau_{1}\right\} .
$$

It is clearly that $s(t)<0$ for $t>0$.

Next we discuss the right free boundary $\Gamma_{+}$. Let $\phi(x, t)=A\left(1-\frac{t}{T-T_{0}}\right) e^{x}$, for $0<t<\tau_{2}$. Then

$$
\begin{gathered}
\phi(x, t)=\varphi(x, t), \quad \text { if } x>0, \\
\phi_{x}(x, t)>0=\varphi_{x}(x, t), \quad \text { if } x<0 .
\end{gathered}
$$

Theorem 5.2. There holds

$$
(u-\varphi)_{x} \leq 0, \text { in }\left\{(x, t) \mid x>0,0<t<\tau_{2}\right\} .
$$

Proof. Setting $w=\phi_{x}-u_{x}$ and $\mathscr{C}=\{(x, t) \mid u(x, t)>\varphi(x, t)\}$, we have

$$
w=\phi_{x}-u_{x}=\phi_{x}-\varphi_{x} \geq 0
$$

for $(x, t) \in Q_{T} \backslash(\mathscr{C} \cup(0,0)), \liminf _{(x, t) \rightarrow(0,0)} w(x, t) \geq 0$ and $\mathscr{L} u_{x}=f_{x}$ in $\mathscr{C}$. We have

$$
\mathscr{L} \phi(x, t)-f=A\left[(\rho-\theta)\left(1-\frac{t}{T-T_{0}}\right)-\frac{1}{T-T_{0}}-\frac{\mu}{a}\right] e^{x} .
$$

Noting that $(\rho-\theta)\left(1-\frac{t}{T-T_{0}}\right)-\frac{1}{T-T_{0}}-\frac{\mu}{a}>0$, when $0<t<\tau_{2}$, we conclude $\mathscr{L} \phi_{x}>f_{x}$. Hence $\mathscr{L} w>0$ in $\mathscr{C}$. Consequently, it follows from Lemma 3.1 that

$$
w=\phi_{x}-u_{x}(x, t) \geq 0, \text { in } \mathscr{C} .
$$

It turns out that $u_{x}(x, t) \leq \varphi_{x}(x, t)$ for $x>0$ and Theorem 5.2 is proved.

Thus, we can set

$$
\sigma(t)=\inf \{x \mid x>0, u(x, t)=\varphi(x, t)\}
$$

where $0<t<\tau_{2}$ and the right free boundary

$$
\Gamma_{+}=\left\{(\sigma(t), t) \mid 0<t<\tau_{2}\right\}
$$


5.2. Continuity of the free boundary. In this part, we shall discuss the continuity of the free boundaries $\Gamma_{-}=\left\{s(t) \mid 0<t<\tau_{1}\right\}$ and $\Gamma_{+}=\left\{\sigma(t) \mid 0<t<\tau_{2}\right\}$.

From (4.5), we see that

$$
\mathscr{L}_{0}(\varphi-u)(x, t)=(\mathscr{L} \varphi-f)(x, t)+\lambda \int_{-\infty}^{+\infty}(\varphi-u)(x+z, t) d N(z)
$$

is $C^{1}(-\infty, 0)$ and strictly decreasing in $x<0$ for fixed $t$. Hence there is a $B(t) \in C^{1}\left[0, \tau_{1}\right)$ such that $\left\{(x, t) \mid \mathscr{L}_{0}(\varphi-u)(x, t)=0\right\}=\{(x, t) \mid x=B(t)\}$. Let

$$
\tilde{\mathscr{P}}^{o}=\left\{(x, t) \mid \mathscr{L}_{0}(\varphi-u)(x, t)>0\right\}=\{(x, t) \mid x<B(t)\} .
$$

The result below is a property for the points on the free boundary.

Theorem 5.3. We have $\Gamma_{-} \subset \tilde{\mathscr{P}}^{o}$. Namely, if $(\bar{x}, \bar{t}) \in \Gamma_{-}$, then $\mathscr{L}_{0}(\varphi-u)(\bar{x}, \bar{t})>0$.

Proof. Observing $\Gamma_{-} \subset \tilde{\mathscr{P}}$, we see that $\mathscr{L}_{0}(\varphi-u)(\bar{x}, \bar{t}) \geq 0$. If $\mathscr{L}_{0}(\varphi-u)(\bar{x}, \bar{t})=0$, then $\bar{x}=B(\bar{t})$ and we will drive a contradiction.

Define the domain $D=\left\{(x, t) \mid B(t)<x<0,0<t<\tau_{1}\right\}$. From Lemma (4.2), it is easily seen that $D \subset \mathscr{C}$. Furthermore, we conclude, noting that $(\bar{x}, \bar{t}) \in \Gamma_{-}$

$$
(u-\varphi)(x, t)>0=(u-\varphi)(\bar{x}, \bar{t}),(x, t) \in D
$$

and

$$
\mathscr{L}_{0}(u-\varphi)(x, t)>0,(x, t) \in D .
$$

We will apply Hopf's lemma for parabolic operator $\mathscr{L}_{0}$ to drive $(u-\varphi)_{x}(\bar{x}, \bar{t})>0$, which is contradicts the fact that $(u-\varphi)_{x}(\bar{x}, \bar{t})=0$ on the free boundary. Domain $D$ may not satisfy the interior ball condition at $(\bar{x}, \bar{t})$. We follow idea in the proof of Lemma 3.2 in [8]. Let $y=x-B(t)$, $\tilde{u}(y, t)=u(x, t)$ and $\tilde{\varphi}(y, t)=\varphi(x, t)$. In the region

$$
\tilde{D}=(y, t) \mid 0<y<-B(t), t \in(0, T]
$$

we have, since $B(t) \in C^{1}(0, T]$

$$
\tilde{\mathscr{L}}_{0}(\tilde{u}-\tilde{\varphi})(y, t)=\mathscr{L}_{0}(u-\varphi)>0
$$

where $\tilde{\mathscr{L}}_{0} u(y, t)=\mathscr{L}_{0} u(y, t)-B^{\prime}(t) u_{y}$. On the other hand, from our assumption, $\bar{y}=\bar{x}-B(\bar{t})=$ 0. Moreover, there clearly exists a ball $\mathscr{B} \subset \tilde{D}$ such that 1$) \mathscr{B} \bigcap\{y=0\}=(0, \bar{t}) ; 2)(\tilde{u}-$ $\tilde{\varphi})(y, t)>(\tilde{u}-\tilde{\varphi})(0, \bar{t})$ for all $(y, t) \in \mathscr{B}$. Now applying Hopf's Lemma (see Theorem 17 in page 49 of Friedman [1964]), we obtain $(u-\varphi)_{x}(\bar{x}, \bar{t})=(\tilde{u}-\tilde{\varphi})_{y}(0, \bar{t})>0$. This contradicts with the fact that $(u-\varphi)_{x}=0$ on the free boundary.

We have the following estimate, which deduces the continuity of the free boundaries.

Lemma 5.1. For any given $0<\delta<\tau_{1}$, there exist constants $C_{\delta}$ and $\varepsilon$ depending only on $\delta$, such that, if $t_{1}, t_{2} \in\left(0, \tau_{1}-\delta\right], s\left(t_{1}\right)<-\delta$ and $0<t_{2}-t_{1}<\varepsilon$, then

$$
s\left(t_{1}\right)-s(t) \leq C_{\delta}\left(t_{2}-t_{1}\right)^{1 / 2}, t_{1}<t<t_{2} .
$$

Proof. Let

$$
E_{\delta}=\left\{(x, t) \mid m\left(\tau_{1}-\delta\right)<x<\min \left\{m(0),-\frac{\delta}{2}\right\}, 0<t<\tau_{1}-\delta\right\}
$$


Then there is a constant $L=L_{\delta}$ depending only on $\delta$ such that $u$ is Lipschitz continuous in $E_{\delta}$ with Lipschitz constant $\frac{L}{2}$. We may assume that $L_{\delta} \geq \frac{A}{T-T_{0}}$ and hence $\varphi$ is Lipschitz continuous in $E_{\delta}$ with Lipschitz constant $\frac{L}{2}$.

Let $\bar{x}=s\left(t_{1}\right)$ and define a domain $D$ by

$$
D:=\left\{(x, t) \mid s(t)<x<\bar{x}, t_{1}<t<t_{2}\right\} .
$$

Obviously, assertion (5.5) holds if $D=\emptyset$. We may hence assume $D \neq \emptyset$. Thus $D$ is an open set. Furthermore, from Theorem 5.3, there exists a constant $\varepsilon$ such that if $0<t_{2}-t_{1}<\varepsilon$, then $\operatorname{dist}\left\{D, \partial \tilde{P}_{-}\right\} \geq d_{\delta}$. Therefore, $\inf _{D} \mathscr{L}_{0}(\varphi-u) \geq c_{\delta}>0$ for positive constants $d_{\delta}, c_{\delta}$ depending only on $\delta$.

Consider the function

$$
w(x)=\beta^{2}\left[(x-X)^{+}\right]^{2},
$$

where $X=\bar{x}-\frac{\sqrt{L\left(t_{2}-t_{1}\right)}}{\beta}, \beta>0$. It is clear that $w \in C^{1,1}(-\infty, \bar{x}]$ and $0 \leq w \leq L\left(t_{2}-t_{1}\right)$. Noting that $(u-\varphi)\left(\bar{x}, t_{1}\right)=0$, we have

$$
|(u-\varphi)(\bar{x}, t)| \leq L\left(t_{2}-t_{1}\right), t_{1} \leq t \leq t_{2}
$$

Hence $w \geq u-\varphi$ if $x=\bar{x}$. Since $u-\varphi=0$ on the rest of $\partial_{p} D$, we have $w \geq u-\varphi$ on $\partial_{p} D$. Furthermore, we deduce that, for $x>X$

$$
\begin{aligned}
\mathscr{L}_{0} w & =-\sigma^{2} \beta^{2}-2 \beta\left(\theta-\lambda k-\frac{\sigma^{2}}{2}\right) w^{\frac{1}{2}}+(\rho+\lambda) w \\
& \geq-\left[\sigma^{2}+\frac{\left(\theta-\lambda k-\frac{\sigma^{2}}{2}\right)^{2}}{\rho+\lambda}\right] \beta^{2} .
\end{aligned}
$$

Choose $\beta$ small enough such that $\mathscr{L}_{0} w \geq-c_{\delta}$. Then $\mathscr{L}_{0} w \geq \mathscr{L}_{0}(u-\varphi)$. By standard maximin principle for $\mathscr{L}_{0}$, we drive $u-\varphi \leq w$ in $D$. We see $w=0$ if $x \leq X$, and $u=\varphi$ if $x \leq X$. Hence

$$
s(t) \geq X=s\left(t_{1}\right)-\frac{\sqrt{L\left(t_{2}-t_{1}\right)}}{\beta}, t \in\left[t_{1}, t_{2}\right] .
$$

This completes the proof of the lemma.

Remark 5.1. Note that our proof is different from the proof of Lemma 3.2 in [8]. There exists a gap in the proof of Lemma 3.2 in [8]: the condition $w-(u-\varphi) \geq 0,(x, t) \in Q_{T} \backslash D$ is not true. Here we apply maximin principle for $\mathscr{L}_{0}$ to avoid the condition $w-(u-\varphi) \geq 0,(x, t) \in Q_{T} \backslash D$.

We next discuss the properties of the free boundary.

Theorem 5.4. Assume (4.10) or (4.11) holds. Then the left free boundary $s(t)$ is a finite-valued continuous function of $t \in\left[0, \tau_{1}\right)$ and satisfies

$$
\begin{aligned}
& s\left(\tau_{1}-0\right)=-\infty, \\
& s(0)=x^{-} \quad \text { in the case of }(4.10), \\
& s(0)=0 \quad \text { in the case of }(4.11) .
\end{aligned}
$$

Proof. Recall the definition of the free boundary $x=s(t)$ :

$$
s(t)=\sup \{x \mid x<0, u(x, t)=\varphi(x, t)\}
$$


and define the set

$$
\mathscr{T}=\left\{t \mid 0<t<\tau_{1}, s(t)>-\infty\right\}=\left\{t \mid 0<t<\tau_{1}, S_{-} \cap(-\infty, 0) \times\{t\} \neq \emptyset\right\} .
$$

From Lemma 4.3, we conclude that $\mathscr{T} \cap\left(0, t_{0}\right) \neq \emptyset$ for any $t_{0} \in\left(0, \tau_{1}\right)$. Combining this with Lemma 5.1 , we can prove that $\mathscr{T}=\left(0, \tau_{1}\right)$. In fact, for any given $0<\delta<\frac{\tau_{1}}{2}$, suppose $t_{1} \in \mathscr{T} \cap\left[\delta, \tau_{1}-\delta\right]$ and $0<t_{2}-t_{1}<\varepsilon$. Then by Lemma 5.1, we have $s(t)>-\infty$ and $t \in \mathscr{T}$ for $t_{1}<t<t_{2}$. Noticing $\varepsilon$ depending only on $\delta$, this implies that if $t_{0} \in \mathscr{T}$, then $t \in \mathscr{T}$ for all $t \in\left[t_{0}, \tau_{1}\right)$. It turns out that $\mathscr{T}=\left(0, \tau_{1}\right)$.

We now claim that $s\left(t_{0}+0\right)$ exists for any $t_{0} \in\left(0, \tau_{1}\right)$. In fact, suppose, otherwise, $s\left(t_{0}+0\right)$ does not exist for some $t_{0} \in\left(0, \tau_{1}\right)$. Then, we have $\bar{t}_{n} \downarrow t_{0}, \tilde{t}_{n} \downarrow t_{0}$ such that

$$
s\left(\bar{t}_{n}\right) \rightarrow M, s\left(\tilde{t}_{n}\right) \rightarrow N, M>N .
$$

Supposing $\bar{t}_{n-1}<\tilde{t}_{n}<\bar{t}_{n}$, by Lemma 5.1 we get

$$
s\left(\tilde{t}_{n}\right)>s\left(\bar{t}_{n-1}\right)-C_{\delta} \sqrt{\bar{t}_{n}-\bar{t}_{n-1}} .
$$

Taking the limit as $n \rightarrow+\infty$ immediately yields $N \geq M$, a contradiction. It is clear that $s\left(t_{0}+\right.$ $0) \geq s\left(t_{0}\right)$ by Lemma 5.1. On the other hand, we have $s\left(t_{0}+0\right) \leq s\left(t_{0}\right)$ from the definition of $s(t)$ and the monotonicity of $u-\varphi$ in $x$. Consequently, $s\left(t_{0}+0\right)=s\left(t_{0}\right)$. Now fix any $t_{0} \in\left(0, \tau_{1}\right)$. In a similar way to the above argument, we can also prove that $s\left(t_{0}-0\right)$ exists and $s\left(t_{0}-0\right) \leq s\left(t_{0}\right)$. If $s\left(t_{0}-0\right)<s\left(t_{0}\right)$, we consider the rectangle

$$
D:=\left\{(x, t): x_{1}<x<x_{2}, t_{0}-\delta<t \leq t_{0}\right\},
$$

where $s\left(t_{0}-0\right)<x_{1}<x_{2}<s\left(t_{0}\right), \delta$ is small enough such that $D \subset\{u>\varphi\}$. Hence $\mathscr{L} u_{x}=f_{x}>$ $0,(x, t) \in D$. By Lemma 5.1, $u_{x}(x, t) \geq 0,(x, t) \in Q_{T}$. But $u_{x}\left(x, t_{0}\right)=\varphi_{x}\left(x, t_{0}\right)=0, x_{1}<x<x_{2}$ which are minimum of $u_{x}$. This is a contradiction. We conclude that $s(t) \in C\left(0, \tau_{1}\right)$.

We next prove that $s\left(0^{+}\right)$exists. Otherwise, by the continuity of $s(t)$ for $t>0$, we may assume $\bar{t}_{n} \downarrow 0, \tilde{t}_{n} \downarrow 0$ such that

$$
s\left(\bar{t}_{n}\right) \rightarrow M, s\left(\tilde{t}_{n}\right) \rightarrow N, N<M<0 .
$$

Supposing $s\left(\tilde{t}_{n}\right), s\left(\bar{t}_{n}\right) \leq \frac{M}{2}$, by Lemma 5.1 with $\delta=-\frac{M}{2}$, we get

$$
s\left(\tilde{t}_{n}\right)>s\left(\bar{t}_{n-1}\right)-C_{\delta} \sqrt{\bar{t}_{n}-\bar{t}_{n-1}} .
$$

Taking the limit as $n \rightarrow+\infty$ yields $N \geq M$, a contradiction. So $s\left(0^{+}\right)$exists and we can define $s(0)=s\left(0^{+}\right)$; then $s(t) \in C\left[0, \tau_{1}\right)$. Set

$$
x_{\omega}= \begin{cases}x^{-}, & \text {in the case of }(4.10), \\ 0, & \text { in the case of }(4.11) .\end{cases}
$$

If $s(0)<x_{\omega}$, then, for $s(0)<x<x_{\omega}$, we get $(u-\varphi)_{t}(x, 0)=(f-\mathscr{L} \varphi)(x, 0)<0$ as in Lemma 4.3 , a contradiction. Thus the assertions (5.7) and (5.8) hold. (5.6) follows from the fact that $s(t)<m(t)$ for $t \in\left(0, \tau_{1}\right)$.

We have the following properties for the right free boundary. 
Theorem 5.5. The free boundary $\sigma(t)$ is a finite-valued continuous function of $t \in\left[0, \tau_{2}\right)$ and satisfies

$$
\begin{aligned}
& \sigma\left(\tau_{2}-0\right)=\infty, \\
& \sigma(0)=x^{+} \text {in the case of }(4.14), \\
& \sigma(0)=0 \text { in the case of }(4.15) .
\end{aligned}
$$

Proof. The proof is analogous to the proof of Theorem 5.4 and is therefore omitted.

\section{Acknowledgments}

The first author was in part supported by NSFC grant number 11371280, and the third author by NSFC grant number U1811461 and by Australian Research Council/Discovery Project DP200100124.

\section{REFERENCES}

[1] M. Sherris, Contigent claims valuation of “Greater of Benefits," Actuarial Reaseach Clearing House. 1 (1993), 87-105.

[2] A. Friedman and W. Shen, A variational inequality approach to financial valuation of retirement benefits based on salary, Finance Stochast. 6 (2002), 272-302.

[3] M. Sherris, The valuation of option features in retirement benefits, Risk Ins. 62 (1995), 509-535.

[4] M. Sherris and W. Shen, Financial valuation of retirement benefits based on salary. In "Quantitative Methods in Finance 1999 Conference," Sydney 1999.

[5] H. Pham, Optimal stopping, free boundary, and American option in a jump-diffusion model, Appl. Math. Optim. 35 (1997), 145-164.

[6] C. Yang, L. Jiang and B. Bian, Free boundary and American options in a jump-diffusion model, European J. Appl. Math. 17 (2006), 95-127.

[7] D. Howison, C. Reisinger and J. H. Witte, The effect of non-smooth payoffs on the penalty approximation of American options, SIAM J. Financial Math. 4 (2013), 539-574.

[8] E. Bayraktar, H. Xing, Analysis of the optimal exercise boundary of American options for jump diffusions, SIAM J. Math. Anal. 41 (2009), 825-860.

[9] Y. Wang, B. Bian, J. Zhang, Viscosity solutions of integro-differential equations and passport options in a jump-diffusion model, J. Optim. Theory Appl. 161 (2014), 122-144.

[10] N. L. Bowers, Jr., H. V. Gerber, J. C. Nickman, D. A. Jones, and C. J. Nesbitt, Actuarial Mathematics, 2nd Ed. Society of Actuaries, Schaumburg, Illinois, 1997.

[11] B. Oksendal, Stochastic Differntial Equations: an Introduction with Applications, World Publishing Corporation, Beijing, 2006.

[12] A. Friedman, Stochastic Differential Equations and Applications, vol. 2. Academic Press, New York, 1976.

[13] A. Friedman, Variational Principles and Free-boundary Problems, Wiley-Interscience Publication, Wiley, New York, 1982. 